\title{
The Safety After Fifty Evaluation trial: Evaluation of the safety and efficacy of antihypertensive therapy with metoprolol in patients 50 to 75 years of age: Study design
}

\author{
Hypertension increases in prevalence with advancing age and is a major risk factor for the \\ development of cerdiovascular disease in elderly patients. However, the presence of coexisting \\ illness, altered drug metabolism, enhanced susceptiblity to drug side effects, and physiologic \\ changes such as reduced plasma volume and lower plasma renin levels make treatment of \\ hypertension in elderly patients more difficult. Nonetheless, several studies have now \\ demonstrated the beneficlal effects of antihypertensive drug therapy in older patients. The \\ Safety After Fifty Evaluation trial was designed to determine the short-term efficacy and \\ tolerabillty of once-daily therapy with the cardioselectlve $\beta$-blocker metoprolol alone or in \\ combination with hydrochlorothiazide in the treatment of mild hypertension in patients $\mathbf{5 0}$ to $\mathbf{7 5}$ \\ years of age. A total of 24,816 patients were enrolled in the trial by 2821 practicing physicians \\ from across the United States. This articie describes the details of the Safety After Fifty \\ Evaluation study design. Results of the trial will be reported separately. (Am HeArT J \\ 1988; 116:301.)
}

Michael W. Rich, MD, Lawrence LaPalio, MD, Anthony Schork, PhD, and the SAFE Coordinators* St. Louis, Mo., Chicago, Ill., and Ann Arbor, Mich.

Data from the National Health Survey conducted from 1960 to 1962 and 1971 to 1974 indicate that among persons 65 to 74 years of age, $35 \%$ of white men, $50 \%$ of black men, $42 \%$ of white women, and $59 \%$ of black women are hypertensive. ${ }^{1,2}$ It has been projected that by the year 2020 the number of people in the United States 65 years or older will exceed 50 million. ${ }^{3}$ Thus the number of elderly persons with hypertension will approach 25 million. ${ }^{4}$

Elevated blood pressure is so common in older persons that until recently it was thought to be a benign consequence of the aging process. It is now known that the influence of hypertension on the

From the Geriatric Cardiology Section, Jewish Hospital at Washington University, the Department of Geriatrics, Loyola-Strick School of Medicine, and the Department of Biostatistics, University of Michigan School of Medicine and School of Public Health.

Supported by a grant from CIBA-GEIGY Corp., Summit, N.J.

Reprint requests: Michael W. Rich, MD, Division of Cardiology, Jewish Hospital of St. Louis, $216 \mathrm{~S}$. Kingshighway, St. Louis, MO 63110.

*Stevo Julius, MD, Charles Tifft, MD, Barry Uretsky, MD, Ronald Brown, MD, Dorothee Perloff, MD, Raymond Magorien, MD, Stephen Glasser. MD, and Robert Wilburn, MD. development of cardiovascular morbidity and mortality is at least as great in the elderly as in the younger population. ${ }^{5}$ For example, in the Framingham Study the risk of cardiovascular disease in persons without other major risk factors increased progressively with rising systolic blood pressure, and the rate of rise was steepest for the oldest cohort ${ }^{5}$ (Fig. 1). The Framingham Study also demonstrated a close correlation between systolic blood pressure and the incidence of stroke ${ }^{6}$ and congestive heart failure. $^{7}$

The treatment of hypertension in the elderly is complicated by a number of factors. A reduction in plasma volume and renin activity ${ }^{8}$ tends to make the elderly hypertensive patient more sensitive to salt restriction and diuretics. A decline in gastrointestinal, hepatic, and renal function results in significant changes in drug metabolism and makes elderly patients more susceptible to side effects from antihypertensive medications. Alterations in the autoregulatory capacity of the central nervous system and baroreceptor responsiveness further predispose elderly patients to adverse drug reactions. Finally, the presence of coexisting illnesses such as coronary 


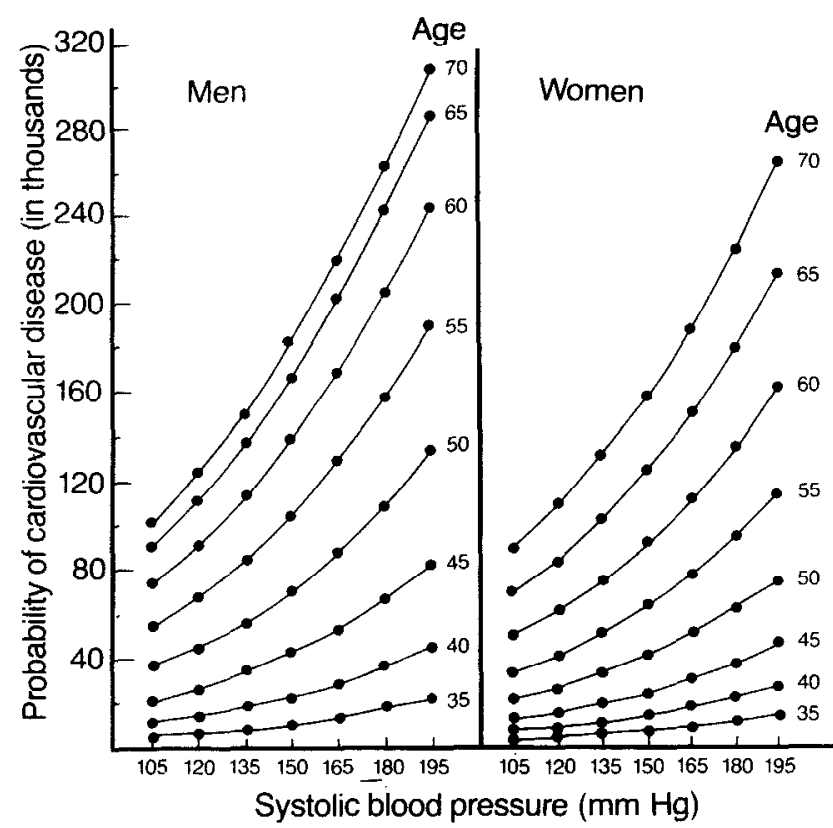

Fig. 1. Probability of developing cardiovascular disease in 8 years, according to systolic blood pressure of low-risk persons 35 to 70 years of age in the Framingham Study. Subjects were persons with serum cholesterol $<185 \mathrm{mg} / \mathrm{dl}$, normal glucose tolerance, no ECG-left ventricular hypertrophy, and nonsmokers. Ordinate shows probability of cardiovascular disease per 1000 persons. (Reproduced from Framingham Monograph, No. 28, Framingham Heart Study.)

heart disease, renal insufficiency, and diabetes may contribute importantly to the selection of an antihypertensive agent for this population.

Despite these difficulties, several studies have now demonstrated a beneficial effect from antihypertensive drug therapy in older patients. In the Veterans Administration Study, ${ }^{9}$ in which about $20 \%$ of patients in the control and treatment groups were age 60 years or above, the incidence of morbid events was substantially reduced by treatment of mild to moderate hypertension (diastolic blood pressure 90 to $114 \mathrm{~mm} \mathrm{Hg}$ ). In the European Working Party on High Blood Pressure in the Elderly trial, ${ }^{10}$ 840 hypertensive patients over the age of 60 years were treated with hydrochlorothiazide and triamterene (methyldopa was also added if needed). After a mean follow-up of 12 years, treated patients experienced a reduction in cardiovascular mortality of $38 \%$, although total mortality was not significantly changed.

Diuneiics have iong veen considered the mainstay of therapy for older patients. Although diuretics have been found to be effective in lowering blood pressure in all ages, there is a greater risk of hypokalemia in older patients, who are more sensitive to the potassium-depleting effects of thiazides than are younger patients. This is of particular importance since hypokalemia may be associated with an increased risk of ventricular arrhythmias and sudden cardiac death. ${ }^{11,12}$ Potassium supplements are often prescribed to alleviate this problem, but older patients are also more prone to hyperkalemia when these agents are used. ${ }^{13}$ Also, some older patients have difficulty following even the simplest of therapeutic regimens; further complicating therapy by the addition of multiple agents reduces the likelihood of compliance. Finally, long-term use of thiazide diuretics leads to a decline in glucose tolerance in most elderly patients, ${ }^{14}$ and uric acid levels also tend to become elevated.

Although it has been suggested that $\beta$-blockers may be less effective in the elderly, they produce significant reductions in blood pressure in most patients. In addition, $\beta$-blockers have the advantage of being effective anti-ischemic and antiarrhythmic agents in the large percentage of elderly patients with clinical or subclinical coronary heart disease.

Recently, an international, multicenter, doubleblind, randomized study was conducted in which hypertensive patients ranging in age from 60 to 75 years were initially treated with once-daily hydrochlorothiazide, $25 \mathrm{mg}$, or metoprolol, $100 \mathrm{mg} .{ }^{15} \mathrm{In}$ patients not responding to hydrochlorothiazide after 4 weeks, the dose was doubled. Metoprolol nonresponders had hydrochlorothiazide, $12.5 \mathrm{mg}$, added to the regimen. Both treatments produced comparable reductions in systolic and diastolic blood pressure. After 4 weeks of therapy, the diastolic blood pressure was reduced to $95 \mathrm{~mm} \mathrm{Hg}$ or less in $50 \%$ of the patients treated with metoprolol and in $47 \%$ of the patients treated with hydrochlorothiazide. After 8 weeks, $65 \%$ of the metoprolol-treated patients and $61 \%$ of the hydrochlorothiazide-treated patients had diastolic blood pressures of $95 \mathrm{~mm} \mathrm{Hg}$ or less. The incidence of side effects was not different between groups, but significantly more patients in the hydrochlorothiazide group had hypokalemia or a rise in uric acid level. Thus, metoprolol and hydrochlorothiazide were equally efficacious, but changes in serum chemistries were more common with the diuretic.

The Safety After Fifty Evaluation (SAFE) study was conducted to evaluate the short-term efficacy and tolerability of once-daily treatment with metoprolol tartrate alone or in combination with a low dose of a thiazide diuretic in patients 50 to 75 years of age with mild hypertension. This multicenter, open-label, phase IV study was designed to include a 
large number of patients treated by their regular physicians in the usual outpatient setting to reflect the realities of clinical practice to the greatest possible extent. Details of the study design will be described; results of the trial will be reported shortly.

\section{MATERIALS AND METHODS}

Patients. Before beginning the study, practicing physicians from across the United States were solicited to participate in the trial. Details of the study protocol and data collection procedures were supplied and all questions were appropriately answered. A total of 2821 physicians participated by enrolling one or more patients.

All patients were 50 to 75 years of age, and it was anticipated that at least $20 \%$ of patients would be older than 60 years. Patients were eligible if they had newly diagnosed hypertension, if hypertension was untreated for at least 3 weeks (such patients were considered "untreated"), or if hypertension was controlled with no more than two drugs. Diastolic blood pressure at entry was 90 to $104 \mathrm{~mm} \mathrm{Hg}$ in untreated patients or $\leq 95 \mathrm{~mm} \mathrm{Hg}$ in treated patients before medication withdrawal. Maximum systolic blood pressure was $200 \mathrm{~mm} \mathrm{Hg}$ for all patients.

Exclusion criteria were angina pectoris requiring $\beta$ blocker therapy, systolic blood pressure $>200 \mathrm{~mm} \mathrm{Hg}$, heart rate $<55$ beats $/ \mathrm{min}$, heart block greater than first degree, congestive heart failure, bronchospastic disease, presence of a cardiac pacemaker, and known intolerance to either $\beta$-blockers or sulfonamides.

Patient enrollment began on Oct. 1, 1986, and continued through Jan. 31, 1987. Follow-up was completed March 28, 1987, and completed patient record forms were accepted through July 31,1987 . The projected enrollment was 30,000 patients $(3000$ physicians $\times 10$ patients per physician); actual enrollment was 24,816 patients ( 8.8 patients per physician). Demographic characteristics of the study population are shown in Table $I$.

Study protocol. At study visit 1 , a detailed medical history was obtained for all patients, with particular emphasis on hypertension history and treatment, presence of other cardiovascular disorders or risk factors, and presence of other major systemic illnesses. A routine physical examination was then performed, which included measurement of the heart rate and systolic and diastolic blood pressure in the sitting and standing positions after a rest period of at least 5 minutes. Blood pressure readings were then repeated after an additional 2 minutes, and the average of the two recordings obtained in the sitting position was used to determine study eligibility.

All patients were initially treated with metoprolol tartrate (Lopressor, Geigy Pharmaceuticals, Summit, N.J.), $100 \mathrm{mg}$ once daily for 4 weeks. Patients then returned for study visit 2 , at which time the heart rate and sitting and standing blood pressure readings were repeated as previously described. A detailed history of any adverse reactions was recorded on a separate study form. Patients with diastolic blood pressure $<90 \mathrm{~mm} \mathrm{Hg}$ at visit 2 were continued on metoprolol, $100 \mathrm{mg}$ once daily. Patients
Table I. Demographic characteristics of study patients

\begin{tabular}{lc} 
Age (yr) & $60 \pm 9$ \\
Male (\%) & 45 \\
Race (\%) & \\
White & 78 \\
Black & 17 \\
Other & 5 \\
Medical history (\%) & \\
Angina pectoris & 12 \\
Prior myocardial infarction & 5 \\
Stroke & 3 \\
Diabetes & 12 \\
Smoking & 19 \\
Baseline heart rate and blood pressure & $80 \pm 10$ \\
Heart rate (beats/min) & $164 \pm 20$ \\
Systolic blood pressure (mm Hg) (sitting) & $95 \pm 9$ \\
Diastolic blood pressure (mm Hg) & \\
$\quad$ (sitting) & \\
\hline
\end{tabular}

with a diastolic pressure $\geq 90 \mathrm{~mm} \mathrm{Hg}$ had hydrochlorothiazide, $25 \mathrm{mg}$, added to their regimen as Lopressor HCT.

This treatment was continued an additional 4 weeks until study visit 3 (study termination). At this time, heart rate, sitting and standing blood pressures, and side effect data were again recorded. Physicians could then continue patients on the study medication, add other agents, or switch to alternative therapy at their discretion.

Data collection and analysis. As each patient completed the study protocol or withdrew from the trial either as a result of side effects or for other reasons, computerized data forms were filled out by the participating physician and forwarded to the core laboratory for statistical analysis at the University of Michigan. Data forms were reviewed fo: completeness, and physicians were contacted as needed to provide supplemental information and ensure that forms were completed for all enrolled subjects.

A total of 24,816 data forms were analyzed. Of these, 13,286 forms ( $54 \%$ ) were complete, meaning that specified study enrollment criteria were fulfilled and all critical data were available from the time the patient entered the study until either the protocol was completed or the patient was withdrawn. Also, data from all patients withdrawn because of documented or suspected adverse drug reactions were analyzed regardless of completeness of the data. Reasons for exclusion from the complete data subset included incomplete data collection $(46 \%)$, age criteria not met $(13 \%)$, blood pressure criteria not met $(20 \%)$, and length of follow-up not within protocol limits $(22 \%)$. The principal statistical analyses were performed for both the entire study population and the complete data subset.

Criteria for efficacy and tolerability. Two major criteria for treatment efficacy were defined as follows: (1) diastolic blood pressure $<90 \mathrm{~mm} \mathrm{Hg}$ and (2) a net decrease in diastolic blood pressure of $>10 \mathrm{~mm} \mathrm{Hg}$. Response rates 
based on either or both of these criteria were determined at study visits 2 and 3 . Tolerability was assessed by analyzing the incidence and significance of all reported side effects. Reasons for all withdrawals and all deaths were analyzed to determine if there was any relationship with the study protocol or medications. At the end of the study, physicians were also asked to subjectively assess each patient's response to therapy with respect to efficacy and tolerability on a 5-point scale ranging from very good to poor. The results of these analyses, as well as subgroup analyses of clinical interest, will be reported separately.

\section{SUMMARY}

Hypertension has a high prevalence in older patients and remains an important risk factor for the development of cardiovascular disease in this population. Treatment of hypertension in the elderly reduces cardiovascular morbidity but is complicated by a number of age-related factors that make selection of an antihypertensive agent more difficult than in younger patients.

The SAFE study was a large clinical trial involving nearly 25,000 patients 50 to 75 years of age with mild hypertension who were treated by their regular physicians in the usual office setting. The purpose of the trial was to assess the short-term safety and efficacy of once-daily therapy with the $\beta$-blocker metoprolol alone or in combination with hydrochlorothiazide in the management of hypertension in these patients. This article summarizes details of the study design and the method of data collection and analysis. The results of this trial, including analysis of major subgroups of clinical interest, will be reported shortly.

\section{REFERENCES}

1. Blood pressure in adults by age and sex, United States 1960-1962. National Health Survey, National Center for
Health Statistics, United States Department of Health, Education, and Welfare, Washington, DC, 1964. (series II, no. 5).

2. Schoenberger JA, Stamler J, Shekelle RB, Shekelle S. Current status of hypertension control in an industrial population. JAMA 1972;222:559-62.

3. U.S. Bureau of the Census, Current population reports, P-25, no. 922. Projection of the population of the United States: 1982-2050 (advance report). Washington, DC: U.S. Government Printing Office, 1982.

4. Messerli FH. Hypertension in the elderly. In: Messerli F, ed. Cardiovascular disease in the elderly. Norwell, Massachusetts: Martinus Nijhoff Publishing, 1984:65-81.

5. Kannel WB, Gordon T. Evaluation of cardiovascular risk in the elderly: the Framingham Study. Bull NY Acad Med 1978;54:573-91.

6. Kannel WB, Wolf PA, Verter J, et al. Epidemiologic assessment of the role of blood pressure in stroke: the Framingham Study. JAMA 1970;214:301-10.

7. Kannel WB, Castelli WP, McNamara PM, et al. Role of blood pressure in the development of congestive heart failure: the Framingham Study. N Fingl J Med 1972;287:781-7.

8. Gavras I, Gavras H, Chobanian AV, et al. Hypertension and age: clinical and biochemical correlates. Clin Exp Hypertens 1982;7:1097-1106.

9. Veterans Administration Cooperative Study on Antihypertensive Agents. Effects of treatment on morbidity in hypertension. II. Results in patients with diastolic blood pressure averaging 90 through $114 \mathrm{~mm} \mathrm{Hg}$. JAMA 1970;213:1143-52.

10. European Working Party on High Blood Pressure in the Elderly (EWPHE). Mortality and morbidity results from the European Working Party on High Blood Pressure in the Elderly Trial. Lancet 1985;1:1349-54.

11. Donnelly T, Gray H, Simpson E, et al. Serum potassium in acute myocardial infarction [Abstract]. Scott Med J 1980; 25:176-.

12. Nordrehaug JE. Malignant arrhythmias in relation to serum potassium values in patients with an acute myocardial infarc. tion. Acta Med Scand 1981;647(suppl):101-7.

13. O'Malley K, O'Brien E. Management of hypertension in the elderly. N Engl J Med 1980;302:1397-1401.

14. Amery A, Berthaux P, Bulpitt C, et al. Glucose intolerance during diuretic therapy: results of trial by the European Working Party on Hypertension in the Elderly. Lancet 1978;1:681-3.

15. Wikstrand J, Westergren G, Berglund G, et al. Antihypertensive treatment with metoprolol or hydrochlorothiazide in patients 60 to 75 years. Report from a double-blind international multicenter study. JAMA 1986;255:1304-10. 